\title{
Response to venepuncture for monitoring in primary schools
}

\author{
Judith Hammond, Susan Chinn, Heather Richardson, Roberto Rona
}

\begin{abstract}
The feasibility and acceptability of collecting blood from children by venepuncture was assessed in a sample of 593 children from seven primary schools in Canterbury. Venepuncture is necessary to obtain blood for the measurement of haemoglobin, ferritin, and cholesterol in line with Department of Health surveys in England.

Return of consent forms was $87 \% ; 75 \%$ of parents in the total sample allowed their child to be tested. Response rates differed between schools. Only 4\% of eligible children refused to participate at the time of testing. In $22(3 \cdot 7 \%)$ children a blood sample could not be obtained or the volume was insufficient for analysis. There was a significant difference in the failure rate between phlebotomists.
\end{abstract}

Venepuncture in the school setting was technically feasible and acceptable. The reluctance of some groups in the community to participate may bias the sample. (Arch Dis Child 1994; 70: 367-372)

Few data have been collected in Britain about the acceptability of blood sampling by venepuncture in surveillance studies of children's health. Venous sampling may be preferable to sampling by fingerprick when the tests to be performed require a large volume of blood. Nevertheless, the acceptability of the procedure to both children and parents may significantly alter response rates to a study and if response rates are less than $65 \%$ the representativeness of the sample, and hence the value of the study, would be brought into question.

In the Dietary and Nutrition Survey of British Adults the response to blood sampling was $65 \%$ of the eligible sample ( $76 \%$ of eligible responders). ${ }^{1}$ In the Bogalusa heart study in the USA, the objective of which was to investigate the early natural history of atherosclerosis, $77 \%$ of children and adolescents provided a blood sample. ${ }^{2}$ The latter study was performed in 1976, before the AIDS scare, and therefore may not represent expected levels of cooperation today. In Britain, a researcher studying a voluntary sample of adolescents in Exeter found high levels of cooperation (nearly $100 \%$ ) in a group of motivated participants. The response rate in a wider sample of voluntary subjects who agreed to participate in the study was only $55 \%$, however, and even lower when the request to collaborate coincided with a high media profile on AIDS (Armstrong N, personal communication).

The acceptability of blood sampling by venepuncture in a paediatric population could be affected by the age of the child, parental attitudes to testing, peer group pressure, or the child's perception of the benefits of participating. The success in collecting the blood sample may depend on the cooperation of the child and the skill of a phlebotomist.

This study was designed to assess whether the introduction of venepuncture into a study of health and growth in primary schoolchildren is a viable option in terms of parental and child consent to participate in the study, and the technical success of collecting blood in the school environment and transporting it safely to a laboratory for analysis. Two strategies of preparing children for the test were compared. In one children were given a short explanation in the classroom as a group before the procedure as well as receiving reassurance from the phlebotomist at the time of testing; the other group were reassured at the time of testing only.

\section{Subjects and methods}

The sample consisted of 593 children. Two hundred and ninety five $(50 \%)$ children were in year 1 (5-6 years of age) and 298 children were in year 4 (8-9 years of age). The sample came from seven schools in Canterbury. With the assistance of the community paediatricians the schools were selected to represent children from a range of socioeconomic backgrounds. The study was carried out to assess the feasibility of venepuncture in schoolchildren, which is necessary to measure haemoglobin, ferritin, and cholesterol concentrations in line with Department of Health surveys in England.

The sample size was chosen to detect a difference in response of $60 \%$ and $75 \%$ between the two strategies of explaining the venepuncture procedure to children, in the two age groups, as significant at the $5 \%$ level with $80 \%$ power.

Approval of the local ethical committee was obtained.

Parents of all the children were sent a letter describing the nature of the study and requesting permission to test their child. A consent 
form was enclosed for the parents to return to the school. Parents were asked to discuss the test with their child before giving consent and were reassured that the child's assent would still be required at the time of testing despite positive parental consent. Parents were also informed that children who underwent venepuncture would be given a $\mathrm{T}$-shirt bearing the study's initials and an illustration of healthy lifestyle as a token of our appreciation of the child's participation and cooperation in the study. Reminders were sent to non-responders one week later.

Three trained phlebotomists were employed to collect the blood samples in the schools.

An explanation before taking a blood sample was given to children in three of the seven schools. Those schools selected included children from varied socioeconomic backgrounds. The explanation was given to all children by one of the investigators $(\mathrm{JH})$ and included a simple description of why the study was being carried out, how the procedure would be performed, and what level of discomfort the children might expect to feel. The children were invited to ask questions about the procedure and their questions were answered. In addition to this explanation, the phlebotomist spent time reassuring the children at the time of testing. In the remainder of the schools the procedure was explained by the phlebotomist at the time of testing only. We did not assess the consistency of the reassurance giving by the phlebotomists to the children.

EMLA anaesthetic cream was applied to children's arms over the proposed venepuncture site one hour before testing (the child returned to class in the interim). Two $5 \mathrm{ml}$ tubes of blood were collected from each child using Vacutainer blood collection sets and tubes (Becton Dickinson) as recommended by the department of clinical biochemistry at the University of Newcastle. The laboratory received no names of children and unused blood was discarded.

The specimens collected were packed into specimen postal containers and sent by first class mail to the laboratory in Newcastle for analysis. This laboratory was suggested by the Department of Health in their efforts to standardise results from a series of health surveys in England. Specimens were only collected between Monday and Thursday to allow at least 24 hours for delivery before the laboratory closing for the weekend on Friday.

\section{ANALYSIS}

Parental consent rates were calculated for individual schools, for the rising 6 and rising 9 year age groups separately, and for the sample as a whole.

Child refusal rates, as a proportion of those for whom parental consent had been obtained and who were not absent from school, were calculated by age group, by type of explanation, and technical failure rates were calculated by the phlebotomists. Response rates between schools, age groups, and type of explanation were compared using $\chi^{2}$ tests and a difference in technical failure rates between phlebotomists was assessed with Fisher's exact test.

The percentage of samples posted which arrived safely and were able to be analysed by the laboratory was also calculated.

\section{Results}

Eighty six per cent of parents completed the consent form (figure). Positive parental consent was obtained from 443 parents, $74.7 \%$ of the total sample. The difference between $72 \%$ consent of year 1 and $77 \%$ of year 4 children was statistically significant $(p<0 \cdot 05)$. Consent was positively refused by 69 parents $(11.6 \%$ of the total sample; $15 \%$ of year 1 and $8 \%$ of year 4 children). The parental positive consent rates for the seven schools participating in the study varied from $46 \%$ to $91 \%$. From the sample of children eligible for testing, $430(97 \cdot 1 \%)$ children presented for testing; $13(2.9 \%)$ children were absent from school during the collection of samples.

Of the 430 children available for testing $21(4.9 \%)$ refused to participate when they arrived at the testing room. This represented $3.5 \%$ of the total sample. The difference between the refusal rates for year 1 and year 4 children was small and not significant. There was no significant difference in refusal rate between those children who received an explanation about the test before entering the testing room and those who received an explanation at the time of testing only.

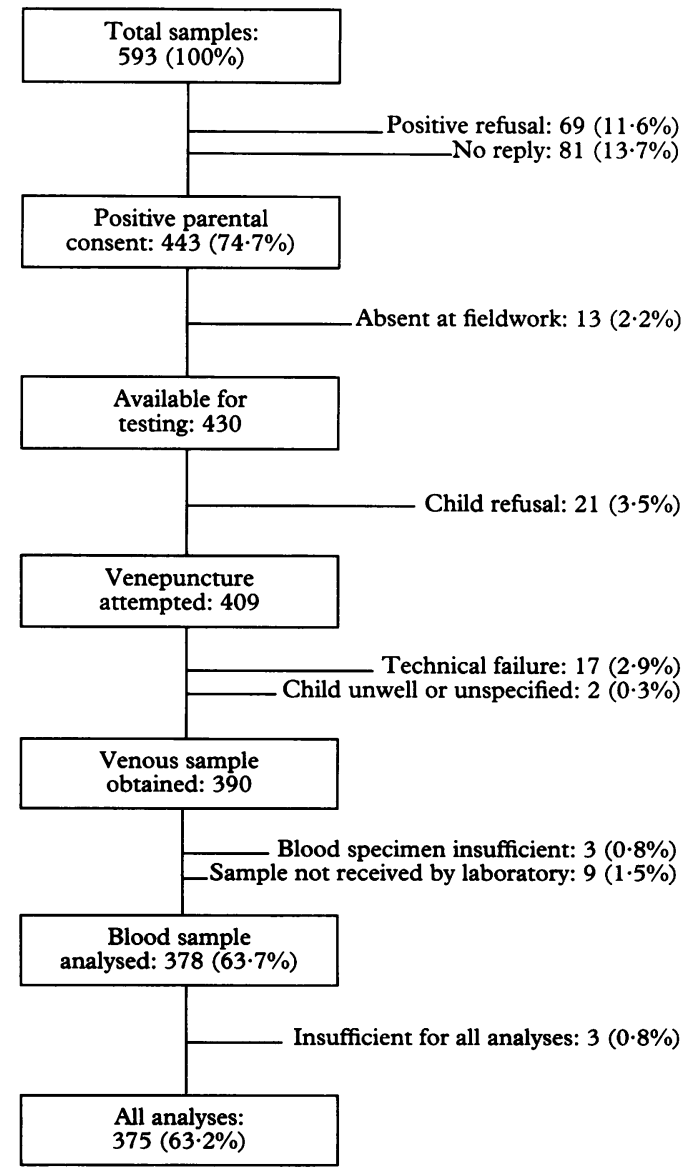

Response to venepuncture. 
Other reasons why blood samples could not be collected included no suitable vein identified (17 (3\%) children) and children feeling unwell at the time of testing (one child). Another child felt unwell but a specimen was collected. Collection of a less than desirable volume of blood was recorded for $17(4 \%)$ children, but 14 of these samples were still analysed. The number of technical failures was none of 279 venepunctures for phlebotomist 1 , one $(1 \%)$ of 67 venepunctures for phlebotomist 2 , and $16(22 \%)$ of 72 venepunctures for phlebotomist 3, the latter having a higher failure rate than either of the other two $(p<0 \cdot 001)$.

Only one child did not have a sample collected without the reason being recorded. Nine samples were reported as collected but were not analysed. Four of these were sent to the wrong address and the fate of the other five samples is unknown. In total there were difficulties in delivering nine $(2 \%)$ of 387 samples from the schools to the laboratory analyser.

A total of 378 blood samples were finally available for analysis, of which 375 were fully analysed.

\section{Discussion}

Overall response rates were satisfactory, though there was a variation in the level of positive consent given between schools. Response rates to a request to collect blood by venepuncture were as high as a previous study by the same workers assessing the response to a request to collect a capillary blood sample from children by fingerprick (unpublished data).

The socioeconomic composition of a school appeared to affect parental response rates. The two schools with the lowest response rates were identified by participating head teachers and local community paediatricians as having larger numbers of children from lower socioeconomic groups and deprived social backgrounds. Conversely, high response rates were obtained from the most middle class schools in the sample.

As found in other studies of invasive testing in children, ${ }^{3}$ response rates in the younger age groups (the rising 6 year age group) were lower than those of the rising 9 year age group. The response rate after the introduction of venepuncture is important as it may have a 'knock on' effect for obtaining consent to the other less invasive tests included in a test battery and affect the overall success of a study.

A small number of parents asked to be present at the time their child was tested. This was allowed, but the venepuncture was often more upsetting for these children than those without parents present. It has been suggested that parents can accurately predict their child's distress associated with painful or anxiety provoking procedures ${ }^{3}$ and parents may wish to be present because they recognise their child's concern. It has also been found that the parent's level of anxiety can influence the child and contribute to the overall apprehension surrounding the procedure. ${ }^{4}$ This is an area that needs further investigation in the context of non-therapeutic research.
Anaesthetic cream has been shown to be highly effective at relieving the pain associated with venepuncture if applied at least one hour before the procedure. ${ }^{5-7}$ Most children appeared to feel minimal, if any, discomfort. The friendly attitudes of the phlebotomists and auxiliary staff and the reassurance that the test was completely voluntary also appeared to be important in minimising children's anxiety.

In this study we have shown that taking blood by venepuncture from even fairly young children in schools for surveillance studies is technically possible. Postal delivery of blood samples to a central laboratory was successful, with minimal loss of specimens. Obtaining positive consent from parents was difficult for some groups of children, raising some concern about the representativeness of the final sample, though the overall response rate obtained in this study was acceptable.

Our recommendations have to be scrutinised in the context of recent guidelines on the conduct of research in children by the Medical Research Council ${ }^{8}$ and British Paediatric Association. ${ }^{9}$ In our view venepuncture is ethical provided that the problem under investigation is of great importance, the response rate is high, and the sample size is appropriate for the question being asked.

We thank Dr M Laker, reader and consultant of the department of clinical biochemistry at the University of Newcastle upon Tyne, Dr K Thida, senior registrar in community paediatrics, Canterbury, and the head teachers and children of the schools who participated in the study for their help and cooperation. The study was funded by the Department of Health.

1 Gregory J, Foster $\mathrm{K}$, Tyler $\mathrm{H}$, Wiseman $\mathrm{M}$. The dietary and nutritional survey of British adults. London: HMSO, 1990.

2 Cresanta JL, Srinivasan SR, Foster TA, Webber LS, Berenson GS. Serum lipoprotein levels in children: epidemiologic and clinical implications. $f$ Chronic Dis 1982; 35: 41-51.

3 Fradet C, McGrath PG, Kay J, Adams S, Luke B. A prospective survey of reactions to blood tests by children and tive survey of reactions to blood

4 Gross AM, Stern RM, Levin RB, Dale J, Wojnilower DA. The effect of mother-child separation on the behaviour of children experiencing a diagnostic medical procedure. f Consult Clin Psychol 1983; 51: 783-5.

5 Hallen R, Olson GL, Uppfeldt A. Pain free venepuncture: effect of timing of application on local anaesthetic cream. Anaesthesia 1984; 39: 969-72.

6 Hellgren U, Kihamia CM, Rombo L. Painless venepuncture in the field. Trans $R$ Soc Trop Med Hyg 1990; 84: 352.

7 Manner T, Kanto J, Lisalo E, Lindberg R, Viinamaki O, Scheinin M. Reduction of pain at venous cannulation in children with eutectic mixture of lidocaine and prilocaine (EMLA cream): comparison with placebo cream and no (EMLA cream): comparison with placebo cream and no

8 Medical Research Council. Working Party on Research in Children. The ethical conduct of research on children. London: MRC, 1991. (MRC Ethics Series.).

9 British Paediatric Association. Guidelines for the ethical conduct of medical research involving children. London: BPA, 1992.

\section{Commentary (1)}

In 1978 the British Paediatric Association (BPA) set up a working party on the ethics of research in children and as a result a set of guidelines to aid ethical committees considering research involving children was published in $1980 .^{1}$ Two of the premises on which the guidelines were based were that (a) research which involves a child and is of no benefit to that child (non-therapeutic research) is not necessarily either unethical or illegal and (b) that the degree of benefit resulting from a research should be assessed in relation to the risk of disturbance, discomfort, or pain - the risk:benefit ratio. 